\title{
UNITED STATES DEPARTMENT OF THE INTERIUR
}

\section{GEOLOGICAL SURVEY}

\author{
Updated Earthquake Calalogue for the States of \\ Florida and Georgia \\ by \\ G. Reagor and C. W. Stover 1
}

Open-File Report $83-823$

This report is preliminary and has not been reviewed for conformity with U.S. Geological Survey editorial standards.

1 Golden, Colorado 


\author{
Updated Eorthauke Catologue for the Stotes of \\ Florida and Georgio \\ G. Reogor and C. W. Stover
}

\title{
INTRODUCTION
}

The data file used to compile the state seismicity maps which were published as a Miscellaneous field Studies series by the U.S. Geological Survey has underoone continual revisions since publication began in 1977. Many of these revisions were based on recent studies of historic earthquakes that provided better estimates of intensity, magnitudes, locations, and source parameters than was given in older literature accounts for a Darticular earthquake. Relocations of historic earthauakes have also had a modifying effect on the data file in the sense the earthquakes are, in some instances, now listed in a different state than the original file.

This report is designed to present the current estimates of epicenter, magnitudes, and intensity for each earthauake in the earthquake catalogue for the States of Florida and Georgia through 1980. The format is similar to the format of the publishea map editions of the U.S. Geological Surver Miscellaneous Field Studies series. This has been done so that the current issues of the published maps may be updated with the changes given in this report. 


\section{CATALOGUE}

The historical earthquake catalogue for the states of florido ond Georgio ore listed in Appendix A ond Appendix B. Prior to 1965 the cotologue contains all felt ond non-felt earthauakes. Subsequent to 1964 restrictions bosed on moanitude ore ploced on the earthquakes 1 isted in the catalogue. Instrumentally recorded earthquakes, which were not known to be felt, ore listed if the magnitude was greater than or equal to 2.5. The earthquake was also included in the catalogue if the earthquoke was felt, regardless of the given magnitude, or if the magnitude was not given. Some aftershocks following large earthauakes are listed but, in many instances, the list of aftershocks is incomplete.

In the explanation of the catalogue, the underlined words correspond to the major headers shown in Appendices $A$ and $B$.

\section{EXPLANATION OF CATALOGUE}

Leaders: (..) indicate information is not available.

RAIE: Date of the earthquake.

QRIGIN IIME: Hour,minute, and second, respectively, in Universal Time (UTC).

LAI: North geographic latitude of the epicenter in degrees.

LONG: Nest geographic longitude of the epicenter in degrees. An asterisk (*) to the right of the longitude indicates the coordinates were assigned by the compiler. An $(x)$ indicates the earthquake is an non-tectonic event.

REPIH: Depth of the earthquake in kilometers.

\section{HYPOCENIER:}

QUAL: Guality of the estimated accuracy of epicenter location.

Instrumental hypocenters are estimated to be accurate within the following degree ranges of latitude ana longitude. Letter-coded range estimates are:

$$
\begin{aligned}
& A=0.0-0.1 \\
& B=0.1-0.2 \\
& C=0.2-0.5 \\
& D=0.5-1.0 \\
& E=1.0 \text { or 1arger. }
\end{aligned}
$$


Non-instrumentol epicenters determined from felt doto ore estimoted to be occurote within the following degree ranges of lotitude and longitude. Letter-coded ronge estimates ore:

$$
\begin{aligned}
& F=0.0-0.5 \\
& G=0.5-1.0 \\
& H=1.0-2.0 \\
& I=2.0 \text { or 1 orger. }
\end{aligned}
$$

REF: Source reference number for the epicenter location (Appendix E).

\section{MAGNIIUDE :}

USGS: USGS mb magnitudes (Gutenbera and Richter, 1956) published in the Preliminary Determination of Epicenters (PDE) by the U.S. Geological Survey, Golden, Colorado and predecessor organzations.

GThEK: Other magnitudes. To the right of the magnitude value is the source code of the contributed maonitude followd by a numerical code which specities ragnitude type.

\section{MAGNITUDE SOURCE CODES}

ATL-Georgia Institute of Technology, At lanta, Ga.

BLA-Virginia Polytechnic Institute ond State University, Blacksturg, Va.

DEn-Dehey, J. W. and Goroon, D.W., 1980, Instrumental seismicity of eastern North America, U.S. Geological Survey (unpublished data).

GB--Bollinger, G. A., 1979, Seismological Society of America Bulletin, v. 69, no. 1, P. 45-63.

GS--National Earthauake Information Service (and Fredecessor organizations), U.S. Geological Survey, Golden, Colo.

JLM-Jones, F. B., Long, L. T., and Mckee, J. H.. 1977, Seismological Society of America Bulletin, v. 67, no. 6. p. 1503-1513.

TAR-Tarr, A. C.. Talwani, Pradeep, Rhea, Susan, Carver, David, and Amick, David, 1981, Results of recent South Carolina seismological studies: Seismological Society of America Bulletin, $v$. 71, no. 6, p. 1883-1902.

MAGNITUDE TYPES 


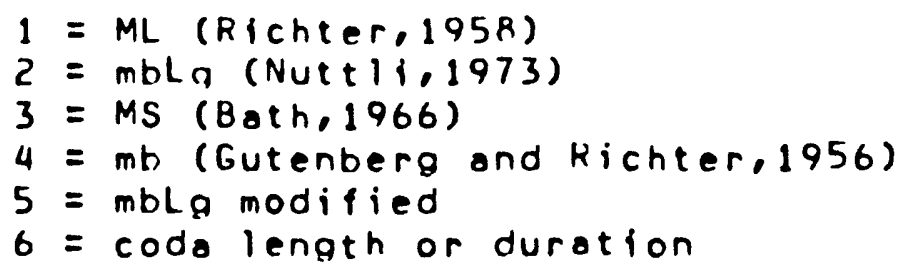

\section{INIENSIIY:}

MN: The maximum Modified Mercalli intensity (niood ond Neumann, 1931) as Roman numerals for the earthauake. An "F" indicates the earthquake was telt, but information was not available to assign an intensity value. An asterisk ( $*$ ) appears to the right of the intensity value if the intensity was assigned by the compiler.

REF: Source reference number of the intensity value (Appendix E). 
The summery of seismicity is listed in Appendix C for florido ond in Appendix D for Georgio. The listings ore on obbrevioted form of the historicol eorthauoke cotologues for the respective states which were used to compile the summaries. The summaries were compiled by rounding the latitude ond longitude to the nearest tenth of a degree. Identical locations were grouped together and counted.

These tabulations, arranged by increasing latitudes, summarize the seismicity according to the maximum intensity experienced at locotions within the two states. Non-tectonic events ore not included in these listings.

The data listed in the summaries are shown in Figures 1 ond 2 on which the plotted numbers or alphabetic characters. representing the maximum intensity volues, are centered at the earthquake coordinates.

To update the published editions of the maps of florida (Stover and others, 1979) and Georgio (Stover ond others, 1979) from the summaries, the year corresponds to the year shown below the triangle: the intensity value corresponds to the value shown to the left of the triangle; and count corresponds to the value shown to the right of the triangle.

In the explanation below, the underlined words correspond to the headers shown in Appendices $C$ and $D$.

\section{EXFLANATION OF APPENDICES C AND D}

Leaders: (..) indicate information is not available.

DAIE: Year,month,day, respectively, of the earthquake which is the most current date for which the maximum intensity was known.

QRIGIN IIME: Origin time in hour, minute, second, respectively, in Universal Time (UTC).

\section{COORRINAIES:}

LAT: North geographic latitude of the epicenter in degrees.

LONG: West geographic longitude of the epicenter in 
degrees. An osterisk ( $(*)$ oppeors to the right of the longltude if the coordinotes were ossigned hy the compiler.

BEE: Quolity of the estimated accuracy of the epicenter followed by the source reference number for the epicenter locotion (Appendix E).

Instrumentol hypocenters are estimated to be accurate within the following degree ranges of latitude ond longitude. Letter-coded range estimates are:

$$
\begin{aligned}
& A=0.0-0.1 \\
& B=0.1-0.2 \\
& C=0.2-0.5 \\
& D=0.5-1.0 \\
& E=1.0 \text { or 1 arger. }
\end{aligned}
$$

Non-instrumental epicenters determined from felt oata are estimated to be accurate within the following degree ranges of latitude and longitude. Letter-coded range estimates are:

$$
\begin{aligned}
& F=0.0-0.5 \\
& G=0.5-1.0 \\
& H=1.0-2.0 \\
& I=2.0 \text { or 1arger. }
\end{aligned}
$$

10: The maximum Modified Mercalli intensity (wood and Neumann, 1931) in arabic numerals at that location. An "F" indicates the earthquake was felt, but information was not available to assign an intensity value. An asterisk ( $*$ ) to the right of the intensity value if the intensity was assigned by the compiler.

IREE: The source reference number for the intensity (Appendix E).

COUNI: The number of earthquakes that occurred at that location. 


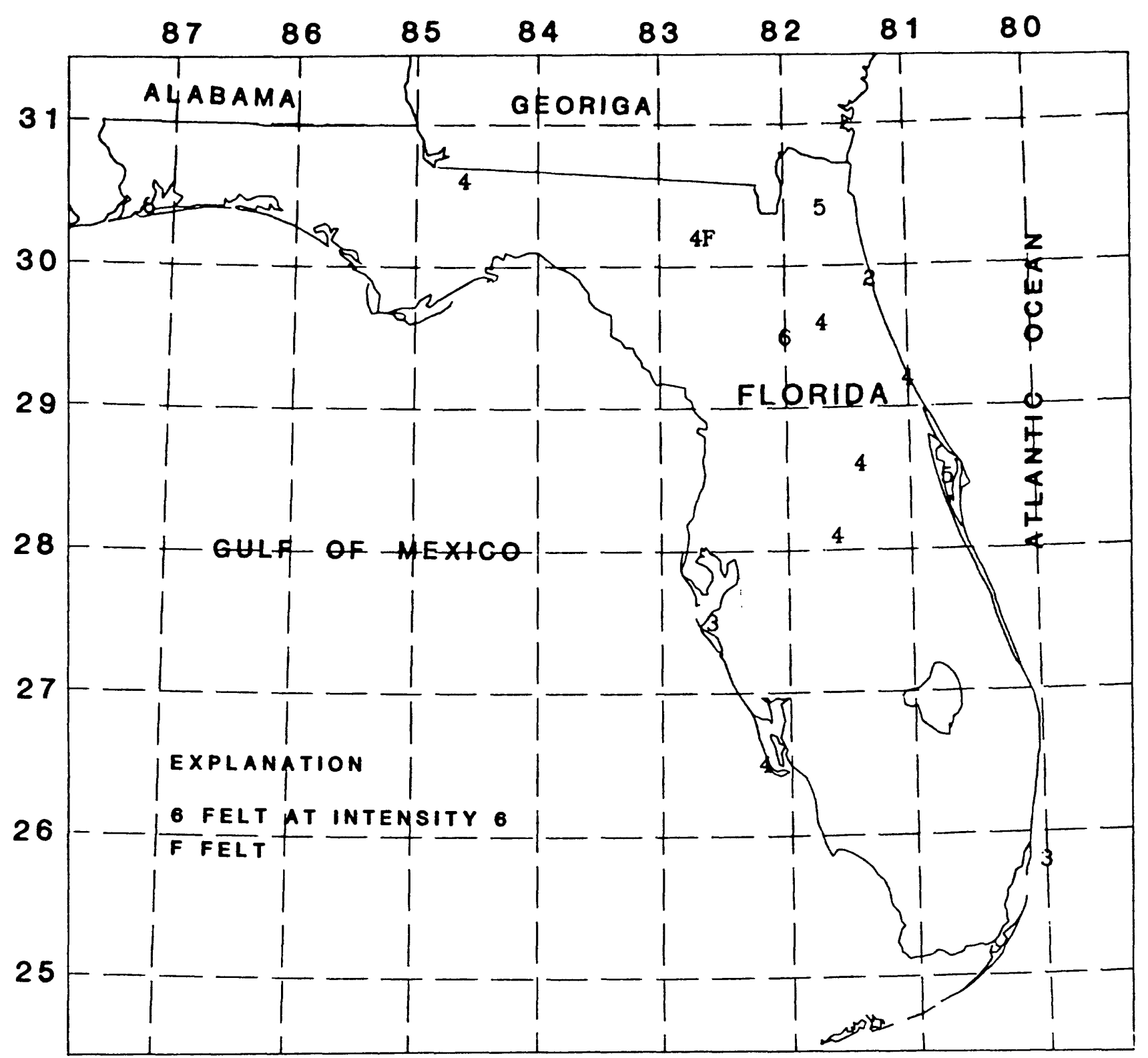

Figure 1.--Maximum Modified Mercalli (MM) intensity experienced at sites in Florida from historical earthquakes, 1780-1980, plotted from Appendix C. Intensities less than 10 are given in arabic numerals. 


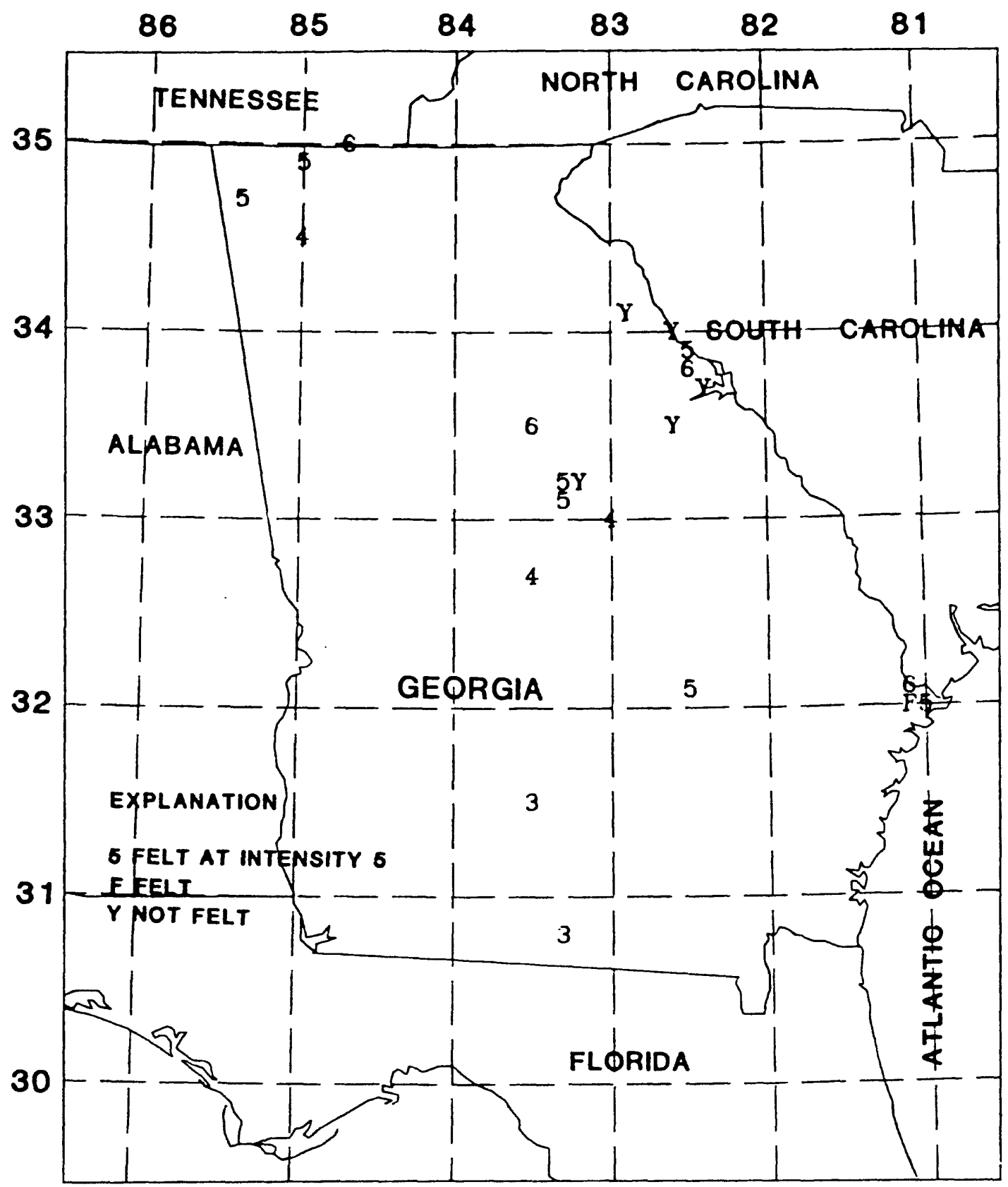

Figure 2.--Maximum Modified Mercalli (MM) intensity experienced at sites in Georgia from historical earthquakes, 1826-1980, plotted from Appendix D. Intensities less than 10 are given in arabic numerals. 
REFERENCES

Both, Markus, 1966, Earthquake energy and magnitude in $v .7$ of Physics and Chemistry of the Earth: Oxford and New York, Peraamon Press, p. 115-165.

Gutenberg, R. and Richter, C. F., 1956, Magnitude and energy of earthquakes: Annuli di Geofisica, v. 9, no. 1, p. 1-15.

Nuttily, 0. W.. 1973, Seismic wave attenuation and magnitude relations for eastern North America: Journal of Geophysics Research, $v .78$, no. 5, p. 876-885.

Richter, C. F., 1958, Elementary Seismology: San Francisco, Calif.. W. H. Freemen and Co.. Inc.. 7680 .

Stover, C. W.. Regor, G.. and Algermissen, S.T., 1979, Seismicity mon of the state of Florida: U.S. Geological Survey Miscellaneous Field Studies Map MF-1056.

Stover, C. W.. Regor, G.. Algermissen, S. T.. and Long, $L$. T.. 1979, Seismicity map of the state of Georgia: U.S. Geological Survey Miscellaneous Field Studies Map MF -1060.

wood, H. O.., and Newman, F., 1931. Modified Mercalli Scale of 1931: Seismological Society of America Bulletin, $v$. 21, no. 4. P. 277-283.

9 
Chronological lleting of earthquakes for the stete of Floride

\begin{tabular}{|c|c|c|c|c|c|c|c|c|c|c|c|c|c|c|c|}
\hline \multirow{2}{*}{$\begin{array}{r}D \\
Y E A R\end{array}$} & \multirow{2}{*}{$\begin{array}{l}\text { A T E } \\
\text { MONTH }\end{array}$} & \multirow{2}{*}{ DAY } & \multicolumn{2}{|c|}{ ORIGIN } & TIME ( UTC) & \multirow{2}{*}{$\begin{array}{l}\text { LAT. } \\
\text { (N.) }\end{array}$} & \multirow{2}{*}{$\begin{array}{l}\text { LONG. } \\
\left(W_{.}\right)\end{array}$} & \multirow{2}{*}{\multicolumn{2}{|c|}{$\begin{array}{l}\text { DEPTH } \\
\text { ( XM) }\end{array}$}} & \multicolumn{2}{|c|}{ HYPOCENTER } & \multicolumn{2}{|c|}{ MAGNITUDE } & \multicolumn{2}{|c|}{ INTENSITY } \\
\hline & & & H & $M$ & $\mathbf{S}$ & & & & & QUAL & REF & USCS & OTHER & MM & REF \\
\hline 1780 & FEB & 06 & $\cdots$ & $\cdots$ & $\ldots$ & 30.4 & 87.2 & $\bullet$ & $\cdots$ & C & 101 & $\cdots$ & $\cdots$ & VI & 101 \\
\hline 1879 & JAN & 13 & 04 & 45 & $\cdots$ & 29.5 & 82.0 & & . & H & 38 & .. & . $\ldots$ & VI & 38 \\
\hline 1879 & JAN & 13 & 04 & SS & .. & 29.5 & 82.0 & & $\cdots$ & $\mathbf{H}$ & 38 & -. & $\cdots \quad \cdots$ & $\boldsymbol{F}$ & 38 \\
\hline 1886 & JAN & 08 & 18 & 34 & $\cdots$ & 30.4 & 81.7 & & . & H & 103 & - & . . & $\mathbf{F}$ & 84 \\
\hline 1886 & SEP & 01 & $\cdots$ & . & . & 30.4 & 81.7 & $\star$ & $\cdots$ & H & 69 & . & $\ldots$ & IV & 69 \\
\hline 1886 & SEP & 03 & 21 & $\cdots$ & $\cdots$ & 30.4 & 81.7 & $\bullet$ & $\cdots$ & H & 84 & $\cdots$ & .. $\cdots$ & IV & 69 \\
\hline 1886 & SEP & 04 & 09 & .. & .. & 30.4 & 81.7 & & .. & H & 103 & . & $\ldots$ & IV & 69 \\
\hline 1886 & SEP & OS & . & .. & .. & 30.4 & 81.7 & $\star$ & .. & и & 69 & .. & .. & IV & 69 \\
\hline 1886 & SEP & 08 & . & .. & .. & 30.4 & 81.7 & $\star$ & .. & H & 69 & .. & . & IV & 69 \\
\hline 1886 & SEP & 09 & 18 & 47 & $\cdots$ & 30.4 & 81.7 & & . & H & 103 & . & $\ldots$ & IV & 69 \\
\hline 1893 & JUN & 21 & 07 & 07 & $\cdots$ & 30.4 & 81.7 & $\star$ & . & H & 84 & .. & $\cdots$ & IV & 69 \\
\hline 1900 & OCT & 10 & $\cdots$ & . & $\cdots$ & 30.3 & 81.7 & $x$ & .. & H & 84 & $\ldots$ & .. & $\mathbf{V}$ & 84 \\
\hline 1900 & OCT & 10 & $\cdots$ & •. & $\cdots$ & 30.3 & 81.7 & $x$ & $\cdots$ & H & 84 & -. & .. & $111 *$ & 84 \\
\hline 1900 & OCT & 10 & $\cdots$ & .. & - & 30.3 & 81.7 & $x$ & .. & H & 84 & .. & $\ldots \quad \ldots$ & III* & 84 \\
\hline 1900 & OCT & 10 & .. & . & $\cdots$ & 30.3 & 81.7 & $x$ & . & H & 84 & $\cdots$ & $\cdots \cdots$ & $I I I *$ & 84 \\
\hline 1900 & $O C T$ & 10 & $\cdots$ & $\cdots$ & $\cdots$ & 30.3 & 81.7 & $x$ & $\cdots$ & H & 84 & - & . & III & 84 \\
\hline 1900 & OCT & 10 & $\cdots$ & .. & . & 30.3 & 81.7 & $x$ & .. & H & 84 & .. & .. & $111 *$ & 84 \\
\hline 1900 & $O C T$ & 10 & $\cdots$ & .. & . & 30.3 & 81.7 & $x$ & -. & H & 84 & .. & .. & III* & 84 \\
\hline 1900 & OCT & 10 & $\cdots$ & . & . & 30.3 & 81.7 & $x$ & . & H & 84 & .. & .. & III* & 84 \\
\hline 1900 & OCT & 31 & 16 & 15 & $\cdots$ & 30.4 & 81.7 & & $\cdots$ & H & 38 & . & $\ldots \ldots$ & v & 38 \\
\hline 1902 & MAY & 21 & $\ddot{m}$ & $\cdots$ & - & 29.9 & 81.3 & $\star$ & $\bullet$ & H & 84 & $\cdots$ & $\cdots$ & $\cdots$ & 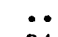 \\
\hline 1902 & MAY & 21 & 00 & . & $\cdots$ & 29.9 & 81.3 & $\star$ & .. & H & 84 & $\cdots$ & $\ldots$ & II & 84 \\
\hline 1905 & SEP & 04 & 09 & 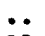 & $\ldots$ & 27.5 & 82.6 & $\star$ & -. & Н & 84 & .. & . & 111 & 84 \\
\hline 1930 & JUL & 19 & 18 & 53 & - & 25.8 & 81.4 & $x$ & $\cdots$ & и & 103 & .. & - & $v$ & 103 \\
\hline 1935 & NOV & 14 & 03 & 10 & $\cdots$ & 29.6 & 81.7 & $\star$ & $\cdots$ & и & 69 & $\cdots$ & $\cdots \cdots$ & IV & 69 \\
\hline 1935 & NOV & 14 & 03 & 30 & $\cdots$ & 29.6 & 81.7 & 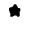 & $\cdots$ & H & 69 & $\cdots$ & $\cdots$ & IV & 69 \\
\hline 1940 & DEC & 27 & 01 & .. & - & 28.0 & 82.5 & $x$ & $\cdots$ & H & 13 & .. & $\cdots$ & $\cdots$ & 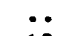 \\
\hline 1942 & JAN & 19 & 19 & .. & .. & 26.5 & 81.0 & $x$ & -. & $I$ & 69 & .. & .. & IV & 69 \\
\hline 1942 & JAN & 19 & • & $\cdots$ & - & 26.5 & 81.0 & $x$ & $\cdots$ & I & 69 & $\cdots$ & $\cdots$ & IV* & 69 \\
\hline 1942 & JAN & 19 & $\bullet$ & $\cdots$ & . & 26.5 & 81.0 & $\mathbf{x}$ & $\cdots$ & I & 69 & . & $\cdots$ & IV* & 69 \\
\hline 1942 & JAN & 19 & . & $\cdots$ & $\cdots$ & 26.5 & 81.0 & $\mathbf{x}$ & -. & I & 69 & . & - & IV* & 69 \\
\hline 1942 & JAN & 19 & -. & - & .. & 26.5 & 81.0 & $\mathbf{x}$ & -. & I & 69 & .. & . & IV* & 69 \\
\hline 1942 & JAN & 19 & .. & $\cdots$ & -. & 26.5 & 81.0 & $x$ & -. & I & 69 & . & $\ldots$ & IV* & 69 \\
\hline 1942 & JAN & 19 & $\cdots$ & $\cdots$ & $\cdots$ & 26.5 & 81.0 & $x$ & $\cdots$ & I & 69 & $\cdots$ & $\cdots \quad \cdots$ & IV* & 69 \\
\hline 1942 & JAN & 19 & $\cdots$ & $\cdots$ & $\cdots$ & 26.5 & 81.0 & $x$ & $\cdots$ & I & 69 & $\cdots$ & $\cdots \cdots$ & IV* & 69 \\
\hline 1942 & JAN & 19 & $\cdots$ & $\cdots$ & $\cdots$ & 26.5 & 81.0 & $\mathbf{x}$ & $\cdots$ & I & 69 & $\cdots$ & $\cdots \quad \cdots$ & IV* & 69 \\
\hline 1942 & JAN & 19 & .. & .. & - & 26.5 & 81.0 & $x$ & $\cdots$ & I & 69 & .. & . & IV* & 69 \\
\hline 1942 & JAN & 19 & $\cdots$ & $\cdots$ & $\cdots$ & 26.5 & 81.0 & $x$ & $\cdots$ & $\mathbf{I}$ & 69 & $\cdots$ & $\cdots \quad \cdots$ & IV* & 69 \\
\hline 1942 & JAN & 19 & $\cdots$ & $\cdots$ & $\cdots$ & 26.5 & 81.0 & $x$ & $\cdots$ & $I$ & 69 & $\cdots$ & $\cdots$ & IV* & 69 \\
\hline 1942 & JAN & 19 & $\cdots$ & $\cdots$ & - & 26.5 & 81.0 & $x$ & $\cdots$ & $I$ & 69 & $\cdots$ & $\cdots \quad \cdots$ & IV* & 69 \\
\hline
\end{tabular}


Chronologicel listing of earthquakes for the state of Florlde (con't.)

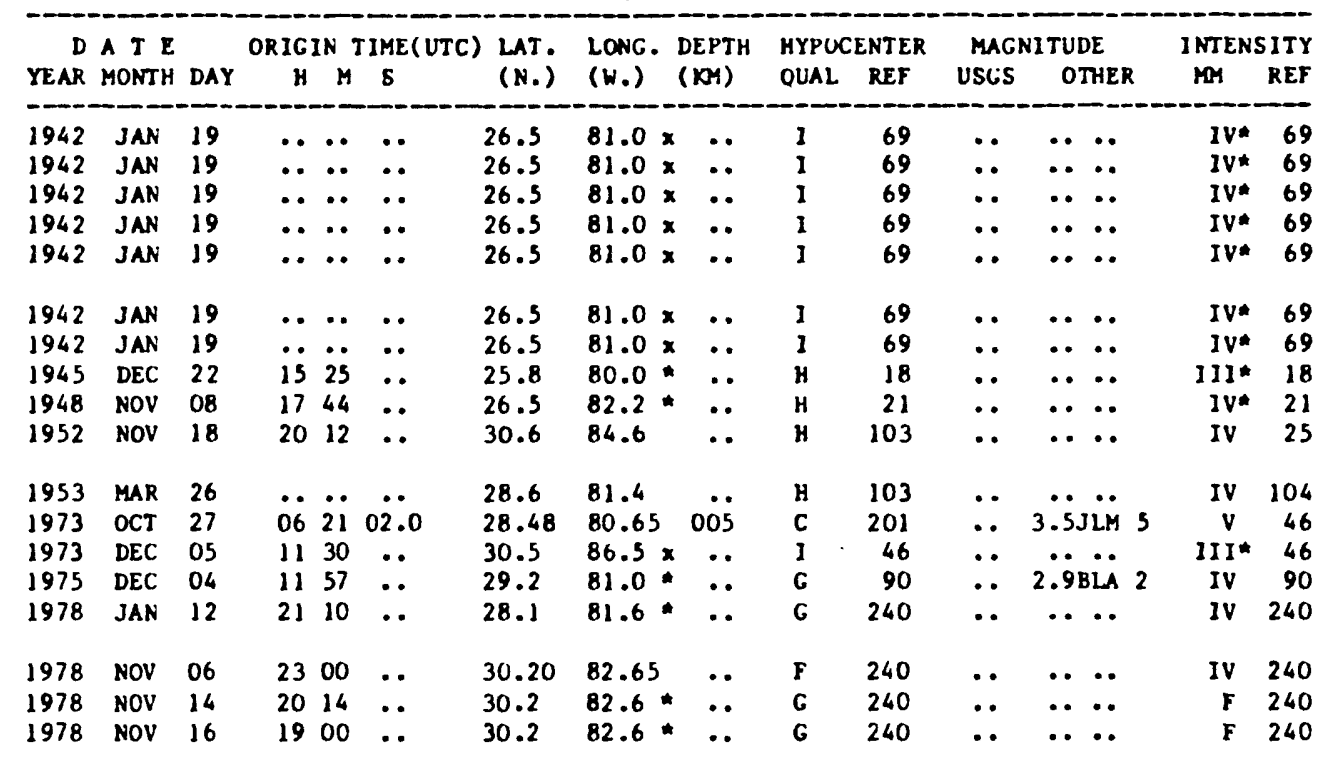


Chronological dieting of earthquaked for the state of Ceorgis

\begin{tabular}{|c|c|c|c|c|c|c|c|c|c|c|c|c|c|c|c|}
\hline & A T $\mathbf{L}$ & & ORICI & IN I & TIME (UTC) & ) LAT. & LONC. & DEPTH & HYPOC & ENTER & MACI & NITUDE & & INTENS & SITY \\
\hline YEAR & MONTH & DAY & H & $M$ & $\mathbf{s}$ & (N.) & $\left(H_{0}\right)$ & (XY) & QUAL & REF & USCS & OTHEF & & $M H$ & REF \\
\hline $\begin{array}{l}1826 \\
1872 \\
1875 \\
1875 \\
1884\end{array}$ & $\begin{array}{l}\text { OCT } \\
\text { JUN } \\
\text { JUL } \\
\text { NOV } \\
\text { MAR }\end{array}$ & $\begin{array}{l}15 \\
17 \\
28 \\
02 \\
31\end{array}$ & $\begin{array}{l}\ddot{20} \\
23 \\
02 \\
10\end{array}$ & $\begin{array}{l}\ddot{00} \\
05 \\
55 \\
00\end{array}$ & $\begin{array}{l}\ddot{ } \\
\ddot{*} \\
\ddot{*}\end{array}$ & $\begin{array}{l}32.0 \\
33.1 \\
33.1 \\
33.8 \\
33.1\end{array}$ & $\begin{array}{l}81.1 \\
83.3 \\
83.3 \\
82.5 \\
83.3\end{array}$ & 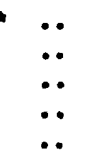 & $\begin{array}{l}\mathbf{H} \\
\mathbf{G} \\
\mathbf{H} \\
\mathbf{G} \\
\mathbf{H}\end{array}$ & $\begin{array}{l}84 \\
38 \\
86 \\
38 \\
86\end{array}$ & $\begin{array}{l}\cdots \\
\cdots \\
\cdots \\
\cdots\end{array}$ & $\begin{array}{ll}\ldots & \cdots \\
\cdots & \cdots \\
\cdots & \cdots \\
\cdots & \cdots\end{array}$ & & $\begin{array}{r}F \\
V \\
I I I \\
V I \\
I I I\end{array}$ & $\begin{array}{l}84 \\
38 \\
86 \\
38 \\
86\end{array}$ \\
\hline $\begin{array}{l}1885 \\
1903 \\
1909 \\
1912 \\
1912\end{array}$ & $\begin{array}{l}\text { OCT } \\
\text { JAN } \\
\text { OCT } \\
\text { JUN } \\
\text { OCT }\end{array}$ & $\begin{array}{l}17 \\
24 \\
08 \\
20 \\
23\end{array}$ & $\begin{array}{l}22 \\
01 \\
10 \\
01\end{array}$ & $\begin{array}{l}30 \\
15 \\
00 \\
10 \\
15\end{array}$ & $\begin{array}{l}\cdots \\
\cdots \\
\ddot{*} \\
\ddot{*}\end{array}$ & $\begin{array}{l}33.0 \\
32.1 \\
34.9 \\
32.0 \\
32.7\end{array}$ & $\begin{array}{l}83.0 \\
81.1 \\
85.0 \\
81.0 \\
83.5\end{array}$ & 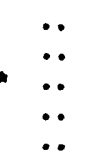 & $\begin{array}{l}\mathbf{H} \\
\mathbf{G} \\
\mathbf{H} \\
\mathbf{H} \\
\mathbf{H}\end{array}$ & $\begin{array}{l}86 \\
38 \\
84 \\
38 \\
84\end{array}$ & $\begin{array}{l}\cdots \\
\cdots \\
\cdots \\
\cdots\end{array}$ & $\begin{array}{ll}\cdots & \cdots \\
\cdots & \cdots \\
\cdots & \cdots \\
\cdots & \cdots\end{array}$ & & $\begin{array}{l}\text { IV } \\
\text { VI } \\
V \star \\
V \\
\text { IV }\end{array}$ & $\begin{array}{l}86 \\
38 \\
84 \\
38 \\
84\end{array}$ \\
\hline $\begin{array}{l}1913 \\
1914 \\
1914 \\
1928 \\
1933\end{array}$ & $\begin{array}{l}\text { MAR } \\
\text { MAR } \\
\text { MAR } \\
\text { MAY } \\
\text { JUN }\end{array}$ & $\begin{array}{l}13 \\
05 \\
05 \\
23 \\
09\end{array}$ & $\begin{array}{l}05 \\
20 \\
21 \\
10 \\
11\end{array}$ & $\begin{array}{l}\ddot{05} \\
00 \\
15 \\
30\end{array}$ & 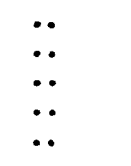 & $\begin{array}{l}34.5 \\
33.5 \\
33.5 \\
30.8 \\
33.3\end{array}$ & $\begin{array}{l}85.0 \\
83.5 \\
83.5 \\
83.3 \\
83.5 \times\end{array}$ & $\begin{array}{l}\cdots \\
\cdots \\
\cdots \\
\cdots\end{array}$ & $\begin{array}{l}1 \\
\mathbf{G} \\
\boldsymbol{F} \\
\mathrm{H} \\
\mathrm{H}\end{array}$ & $\begin{array}{r}103 \\
38 \\
289 \\
1 \\
86\end{array}$ & $\begin{array}{l}\cdots \\
\cdots \\
\cdots \\
\cdots\end{array}$ & $\begin{array}{ll}\ldots & \cdots \\
\ldots & \ldots \\
\ldots & \ldots \\
\ldots & \cdots \\
\ldots & \cdots\end{array}$ & & $\begin{array}{l}\text { IV } \\
\text { VI } \\
\ddot{I I I} \\
\text { IV }\end{array}$ & $\begin{array}{r}103 \\
38 \\
\cdots \\
1 \\
102\end{array}$ \\
\hline $\begin{array}{l}1943 \\
1958 \\
1963 \\
1964 \\
1964\end{array}$ & $\begin{array}{l}\text { JUL } \\
\text { APR } \\
\text { OCT } \\
\text { FEB } \\
\text { FEB }\end{array}$ & $\begin{array}{l}29 \\
08 \\
08 \\
17 \\
18\end{array}$ & $\begin{array}{l}03 \\
17 \\
06 \\
22 \\
09\end{array}$ & $\begin{array}{l}30 \\
01 \\
47 \\
31\end{array}$ & $\begin{array}{c}\ddot{0} \\
4 \ddot{3} .4 \\
\ddot{10.4}\end{array}$ & $\begin{array}{l}33.4 \\
31.5 \\
33.9 \\
34.7 \\
34.67\end{array}$ & $\begin{array}{l}82.0 \times \\
83.5 \\
82.5 \\
85.4 \\
85.39\end{array}$ & $\begin{array}{c}\ldots \\
\ldots \\
\ddot{0}\end{array}$ & $\begin{array}{l}H \\
H \\
C \\
D \\
\text { A }\end{array}$ & $\begin{array}{r}16 \\
29 \\
110 \\
203 \\
201\end{array}$ & $\begin{array}{c}\ldots \\
\ldots \\
\ddot{4} \\
4.4\end{array}$ & $\begin{array}{l}\cdots \\
\cdots \\
3.2 \mathrm{JLM} \\
3.3 \mathrm{JLM} \\
4.0 \mathrm{OB}\end{array}$ & $\begin{array}{l}5 \\
5 \\
2\end{array}$ & $\begin{array}{l}I I I * \\
I I I * \\
\cdots \\
\cdots \\
V\end{array}$ & $\begin{array}{l}16 \\
29 \\
\ldots \\
\ldots \\
35\end{array}$ \\
\hline $\begin{array}{l}1964 \\
1964 \\
1965 \\
1965 \\
1965\end{array}$ & $\begin{array}{l}\text { MAR } \\
\text { MAR } \\
\text { APR } \\
\text { JUL } \\
\text { NOV }\end{array}$ & $\begin{array}{l}07 \\
13 \\
07 \\
22 \\
08\end{array}$ & $\begin{array}{l}18 \\
01 \\
07 \\
23 \\
12\end{array}$ & $\begin{array}{l}02 \\
20 \\
41 \\
55 \\
58\end{array}$ & $\begin{array}{l}58.6 \\
17.5 \\
10.2 \\
33.3 \\
01.0\end{array}$ & $\begin{array}{l}33.72 \\
33.19 \\
33.9 \\
33.2 \\
33.2\end{array}$ & $\begin{array}{l}82.39 \\
83.31 \\
82.5 \\
83.2 \\
83.2\end{array}$ & $\begin{array}{c}005 \\
001 \\
\ldots \\
\ldots \\
\ldots\end{array}$ & $\begin{array}{l}B \\
B \\
C \\
C \\
C\end{array}$ & $\begin{array}{l}201 \\
201 \\
110 \\
115 \\
115\end{array}$ & 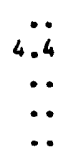 & $\begin{array}{c}3.3 \mathrm{JLM} \\
3.9 \mathrm{JLM} \\
\ldots \quad \ldots \\
3.3 \mathrm{JLM}\end{array}$ & $\begin{array}{l}5 \\
5\end{array}$ & $\begin{array}{c}\cdots \\
v \\
\cdots \\
\cdots \\
\cdots\end{array}$ & $\begin{array}{l}35 \\
\ldots \\
\ldots \\
\ldots\end{array}$ \\
\hline $\begin{array}{l}1965 \\
1969 \\
1969 \\
1969 \\
1969\end{array}$ & $\begin{array}{l}\text { NOV } \\
\text { MAY } \\
\text { MAY } \\
\text { MAY } \\
\text { NOV }\end{array}$ & $\begin{array}{l}08 \\
05 \\
09 \\
18 \\
04\end{array}$ & $\begin{array}{l}13 \\
17 \\
\ldots \\
18\end{array}$ & $\begin{array}{l}04 \\
14 \\
\ldots \\
\ldots 8\end{array}$ & $\begin{array}{c}11.5 \\
\ldots \\
\ldots \\
23\end{array}$ & $\begin{array}{l}33.2 \\
33.9 \\
33.95 \\
33.95 \\
33.2\end{array}$ & $\begin{array}{l}83.2 \\
82.5 \\
82.58 \\
82.58 \\
83.2\end{array}$ & $\begin{array}{l}\cdots \\
\cdots \\
\cdots \\
\cdots\end{array}$ & $\begin{array}{l}\text { C } \\
\text { H } \\
\text { B } \\
\text { F } \\
\text { C }\end{array}$ & $\begin{array}{r}115 \\
86 \\
164 \\
164 \\
115\end{array}$ & $\begin{array}{l}\cdots \\
\cdots \\
\cdots \\
\cdots\end{array}$ & $\begin{array}{c}\ldots \\
\cdots \\
3.3 A T L \\
3.5 A T L \\
\ldots\end{array}$ & $\begin{array}{l}2 \\
2\end{array}$ & $\begin{array}{l}\cdots \\
\cdots \\
\cdots \\
\cdots \\
\cdots\end{array}$ & $\begin{array}{l}\cdots \\
\ldots \\
\ldots \\
\cdots\end{array}$ \\
\hline $\begin{array}{l}1969 \\
1971 \\
1973 \\
1974\end{array}$ & $\begin{array}{l}\text { NOV } \\
\text { APR } \\
\text { OCT } \\
\text { AUG }\end{array}$ & $\begin{array}{l}O B \\
16 \\
08 \\
02\end{array}$ & $\begin{array}{l}01 \\
07 \\
13 \\
08\end{array}$ & $\begin{array}{l}52 \\
31 \\
38 \\
52\end{array}$ & 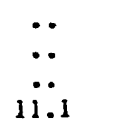 & $\begin{array}{l}33.9 \\
33.9 \\
33.9 \\
33.91\end{array}$ & $\begin{array}{l}82.5 \\
82.5 \\
82.5 \\
82.53\end{array}$ & 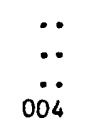 & $\begin{array}{l}\text { C } \\
B \\
B \\
A\end{array}$ & $\begin{array}{l}115 \\
110 \\
110 \\
201\end{array}$ & $\begin{array}{l}\ddot{ } \\
\ddot{4} \\
4.3\end{array}$ & $\begin{array}{l}\ldots \\
\ldots \\
\ddot{4} \dot{1} \dot{\mathrm{GB}}\end{array}$ & 2 & $\begin{array}{l}\cdots \\
\ddot{*} \\
\dot{v}\end{array}$ & $\begin{array}{l}\ddot{ } \\
\ddot{4}\end{array}$ \\
\hline $\begin{array}{l}1975 \\
1976 \\
1976 \\
1978 \\
1980\end{array}$ & $\begin{array}{l}\text { APR } \\
\text { FEB } \\
\text { DEC } \\
\text { JUN } \\
\text { SEP }\end{array}$ & $\begin{array}{l}01 \\
04 \\
27 \\
05 \\
10\end{array}$ & $\begin{array}{l}21 \\
19 \\
06 \\
21 \\
19\end{array}$ & $\begin{array}{l}09 \\
53 \\
57 \\
37 \\
49\end{array}$ & $\begin{array}{l}10 \\
53.0 \\
15.2 \\
44.6 \\
46.4\end{array}$ & $\begin{array}{l}33.2 \\
34.97 \\
32.06 \\
33.54 \\
34.12\end{array}$ & $\begin{array}{l}83.2 \\
84.70 \\
82.50 \\
82.59 \\
82.94\end{array}$ & $\begin{array}{l}\ldots 1 \\
014 \\
014 \\
023 \\
013\end{array}$ & $\begin{array}{l}\mathrm{D} \\
\mathrm{A} \\
\mathrm{A} \\
\mathrm{A} \\
\mathrm{B}\end{array}$ & $\begin{array}{l}203 \\
201 \\
201 \\
290 \\
322\end{array}$ & $\begin{array}{l}\cdots \\
\cdots \\
\cdots \\
\cdots\end{array}$ & $\begin{array}{l}3.9 \mathrm{JLM} \\
3.6 \mathrm{DEW} \\
3.7 \mathrm{BLA} \\
2.6 \mathrm{TAR} \\
2.5 \mathrm{GS}\end{array}$ & $\begin{array}{l}5 \\
2 \\
2 \\
6 \\
6\end{array}$ & $\begin{array}{r}\ddot{v i} \\
v \\
\ldots \\
\ldots\end{array}$ & $\begin{array}{l}\ldots 9 \\
49 \\
\ldots \\
\ldots\end{array}$ \\
\hline
\end{tabular}


Append1x C

Summary of selsmicity - State of Florida

\begin{tabular}{|c|c|c|c|c|c|c|c|c|c|c|c|}
\hline \multirow{2}{*}{$\begin{array}{c}\text { D A } \\
\text { YEAR }\end{array}$} & \multirow{2}{*}{$\begin{array}{l}\mathrm{A} \\
\mathrm{MO}\end{array}$} & \multirow{2}{*}{$\begin{array}{l}\mathbf{E} \\
\mathrm{DA}\end{array}$} & \multicolumn{2}{|c|}{ ORIGIN } & \multirow{2}{*}{)$^{\text {TIME }}$} & \multicolumn{2}{|c|}{ COORDINATES } & \multirow[t]{2}{*}{ REF } & \multirow[t]{2}{*}{ I0 } & \multirow[t]{2}{*}{ IREF } & \multirow[t]{2}{*}{ COUNT } \\
\hline & & & & (UTC & & LAT. & LON. & & & & \\
\hline 1945 & 12 & 22 & 15 & 25 & - & $25.8 N$ & $80.0 W^{\star}$ & H 18 & $3 *$ & 18 & \\
\hline 1948 & 11 & 08 & 17 & 44 & . & $26.5 \mathrm{~N}$ & $82.2 W^{\star}$ & H 21 & $4 \star$ & 21 & \\
\hline 1905 & 09 & 04 & 09 & . & -. & $27.5 \mathrm{~N}$ & $82.6 W^{*}$ & H 84 & 3 & 84 & \\
\hline 1978 & 01 & 12 & 21 & 10 & - & $28.1 \mathrm{~N}$ & $81.6 W^{\star}$ & G240 & 4 & 240 & \\
\hline 1973 & 10 & 27 & 06 & 21 & 02.0 & $28.5 \mathrm{~N}$ & $80.7 W$ & $\mathrm{C} 201$ & 5 & 46 & \\
\hline 1953 & 03 & 26 & $\cdots$ & 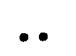 & - & 28.6 & $81.4 W$ & H 103 & 4 & 104 & \\
\hline 1975 & 12 & 04 & 11 & 57 & . & $29.2 \mathrm{~N}$ & $81.0 W^{*}$ & G 90 & 4 & 90 & \\
\hline 1879 & 01 & 13 & 04 & 45 & .. & $29.5 \mathrm{~N}$ & $82.0 W$ & H 38 & 6 & 38 & \\
\hline 1935 & 11 & 14 & 03 & 30 & - & $29.6 \mathrm{~N}$ & $81.7 W^{\star}$ & H 69 & 4 & 69 & \\
\hline 1902 & 05 & 21 & 00 & . & - & $29.9 \mathrm{~N}$ & $81.3 W^{\star}$ & H 84 & 2 & 84 & \\
\hline 1978 & 11 & 16 & 19 & 00 & .. & $30.2 \mathrm{~N}$ & $82.6 W^{\star}$ & G240 & F & 240 & \\
\hline 1978 & 11 & 06 & 23 & 00 & .. & $30.2 \mathrm{~N}$ & $82.7 \mathrm{~W}$ & F240 & 4 & 240 & \\
\hline 1900 & 10 & 31 & 16 & 15 & - & $30.4 \mathrm{~N}$ & $81.7 W$ & H 38 & 5 & 38 & \\
\hline 1780 & 02 & 06 & .. & & .. & $30.4 \mathrm{~N}$ & $87.2 W^{\star}$ & G101 & $6 \star$ & 101 & \\
\hline 1952 & 11 & 18 & 20 & 12 & .. & $30.6 \mathrm{~N}$ & $84.6 \mathrm{~W}$ & H103 & 4 & 25 & \\
\hline
\end{tabular}


Summary of selsmicity - State of Georgla

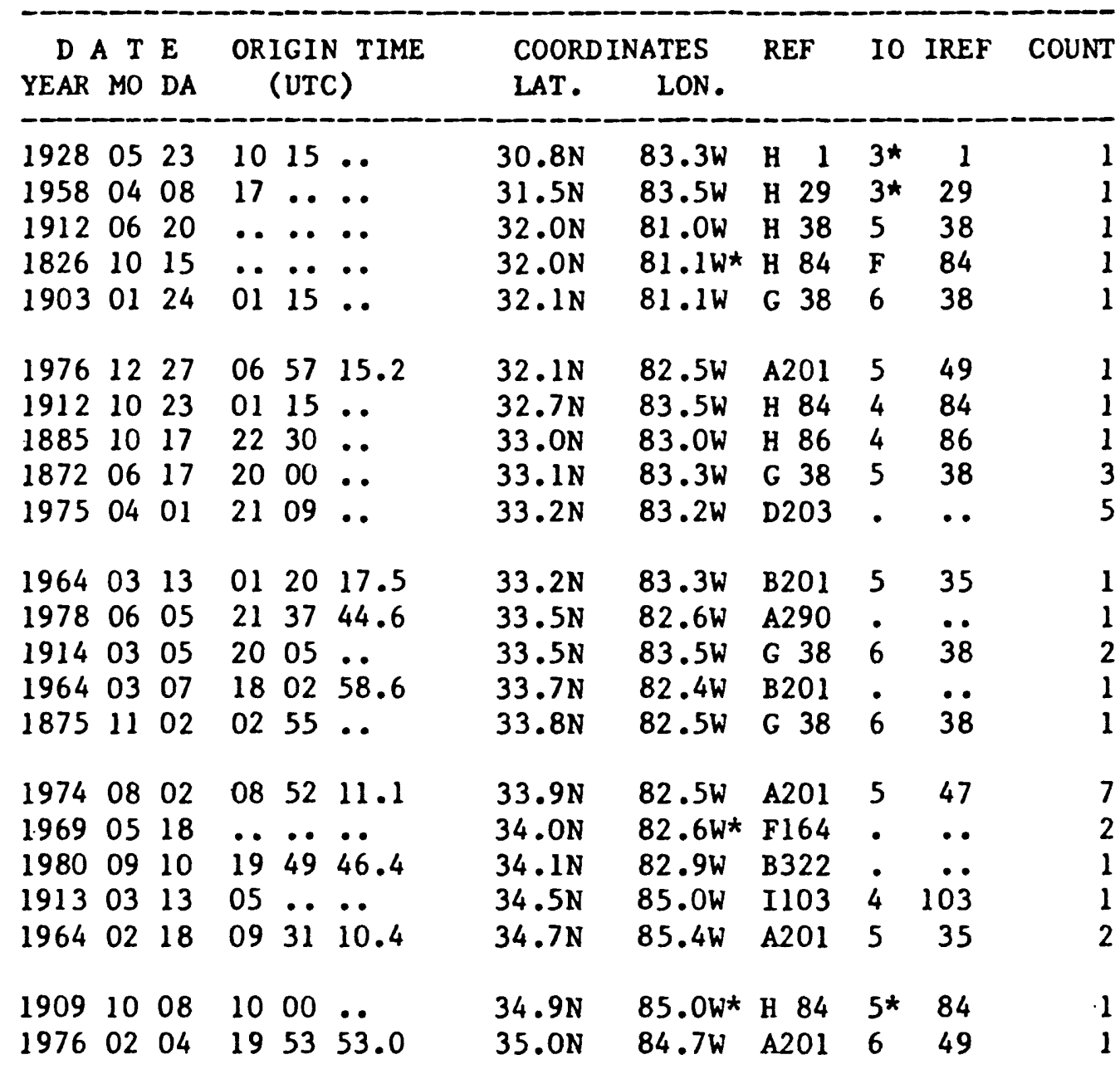


1.--Heck, N. H., and bodle, R. R., 1930, United Stotes Eorthquakes 1928: U. S. Coast ond Geodetic Survey, Seriol No. $483,28 \mathrm{p}$.

3.--Neumann, Frank, and Bodle, R. R., 1932, United States Eorthavakes 1930: U. S. Coast and Geodetic Survey. Serial No. 539, 25 p.

13.--Neumann, Frank, 1942, United States Earthquakes 1940: U. S. Coast and Geodetic Survey, Serial No. 647, 74 p.

16.--Bodle, R. R., 1945, United States Earthquakes 1943: U. S. Coast and Geodetic Survey, Serial No. 672, 47 p.

18.--Bodle, R. R.. ana Murphy, L. M.. 1947, United States Earthauakes 1945: U. S. Coost and Geodetic Survey, Serial No. 699,38 p.

21.--Murphy, L. M., and Ulrich, F. P.. 1951, United States Earthquakes 1948: U. S. Coast and Geodetic Survey, Serial No. 746,50 p.

25.--Murohy, L. M.., and Cloud, W. K.. 1954, United states Earthquakes 1952: U. S. Coast and Geodetic Survey, Serial No. $773,112 \mathrm{p}$.

29.--Brazee, R. J.. and Cloud, W. K., 1958, United States Earthquakes 1956: U. S. Coast and Geodetic Survey, 78 D.

35.--Lander, J. F., and Cloud, W. K., 1904, United States Earthauakes 1962: U. S. Coast and Geodetic Survey, 114 p.

38.--Coffman, J. L., and von Hake, C. A., 1973, Earthquake history of the United States: U. S. National Oceanic and Atmospheric Administration. No. 41-1(through 1970), 208 p.

46.--Coffman, J. L., von Hake, C. A.. Spence, M.., Carver, D. L., Covington, P. A.. Dunphy, G. J.., Irby, W. L.., Person, W. J., and Stover, C. W., 1975, United States Earthauakes 1973: U. S. National Oceanic and Atmospheric Administration and $U$. S. Geological Survey. $112 \mathrm{Ne}$

47.--Coffman, J. L., and Stover, C. W., 1976, United States Earthquakes 1974: U. S. National Oceanic and Atmospheric Administration and $U$. S. Geological Survey, $135 \mathrm{p}$. 
49.--Coffman, J. L., and Stover, C. W.. 1978, United Stotes Earthquakes 1976: U. S. Notionol Uceanic ond Atmospheric Administrotion and $U, S$. Geologicol Survey. $94 \mathrm{p}$.

69.--Campbell, R. B., 1942, Earthquokes in Florido: Florida Acodemy of Science Prodeedings, $v .6$, no. 1 , p. 1-4.

84.--woollard, G. P.. 1968, A catalogue of earthquakes in the United states prior to 1925 based on unpublished data compiled by Harry Fielding Reid and unpublished sources prior to 1930: Hawaij Institute of Geophysics, University of Hawai, Data Report No. 10.

86.--Bollinger, G. A., 1975, A catalogue of southeastern Uniteo States earthauakes 1754 through 1974: Department of geological Science, Virginia Polvtechnic Institute ano State University, Research Bulletin 101, 68p.

90.--Minsch, J. H.. Stover, C. K.. Person, W. J.. ana Simon, K. B.. 1977, Earthquakes in the United States, Uctober-December 1975: U. S. Geological Survey Circular $749-0,27$.

101.-Campbell, R. L., 1975, Historical sketches of colonial Florida: A facsimile reproduction of the 1892 edition, A University of Florida Book. The University of Florida Press, Gainsville, Fl.

102.-Georgia Power Company, 1968, Part II, section B of Preliminary Safety Analysis Report, Edwin A. Hatch nuclear power plant, Unit 1, Docket No. 50-231, Nuclear Regulatory Commission, Public Documents Room, P. A2-36A. $2-41$.

103.-McClain, h. C.. and Meyers, O. M.. 1970, Seismic history and seismicity of the southeastern region ot the Uniteo States: Dak Ridge National Laboratory, Oak Ridge, Tenn.. Union Carbide Corp.. for the U. S. Atomic Energy Commission, P. 1-43.

104.-Seismological Society of America, 1953, Seismological Notes, Seismological Society of America Bulletin, v. 43, no. 2 , p. 179-189.

110.-Denman, H. E.. Jr., 1974, Implications of seismic activity at the Clark Hill Reservoir, Masters Thesis, Georgia Institute of Technology, $103 \mathrm{p}$.

115.-Long, L. T.. 1979, Summary of the historical seismicity of the hallace Dam area, attachment to the quarterly report on seismic monitoring, Georgia Institute of Technology. 
164.-Tolwonl, Prodeep, Secor, D. T., ond Scheffler, P. K.. 197b, Preliminary results of oftershock studies following the 2 August 1974 South Corolino eorthquake: Forthquoke Notes, Eostern Secion, Seismologicol Society of America, $v .46$, no. $4, p .21-28$.

201.-Dewey, J. N., and Gordon, n. W.. 1983, Seismicity of the eastern United States and odjacent Canado: $U$. S. Geological Survey Frofessional Paper $\star \star \star, ~ 105$ p.

203.-Jones, F. E., Lona, L. T., and Mckee, J. H., 1977, Study of the attenuation and azimuthal depenoence of seismic wave propagation in the southeastern United States: Seismological Society of Americal Bulletin. v. 67, no. b. p.1503-1513.

240.-Stover, C. M., and von Hake, C. A., 1980, United States Earthquakes 1978: U. S. Geological Survey and U. S. National Ciceanic and Atmospheric Administration, $112 \mathrm{p}$.

289.-Visvanathan, T. R., 1980. Earthauakes in South Carolina 1696-1975: South Carolina Geological Survey, Bulletin 40 , $61 p$.

290.-Tarr, A. C.. Talwani, Pradeep, Rhea, Susan, Carver, Davia and Amick, Cavid, 1981. Fesults of recent South Carolina seismological studies: Seismological Society of America Bulletin, v. 71, no.6, p. 1883-1902.

322.-Bollinger, G. A.. and Sibol, M. S., 1983, Listing of hyoocenters from Southeastern Seismic Network: Department of Geclogical Sciences, Virginia Polytechnic ano State University, Bulletin no. 10A, 38 P. 